\title{
LA SATIRE DE LA FEMME DANS LE THÉÂTRE DE GeORGES COURTELINE
}

\author{
Recherche de magistère \\ Présenté par \\ Hebatulah Ibrahim Abdel Moneim \\ Assistante au départment de français \\ Faculté des Lettres de Mansoura
}

Sous la direction de:

Dr. Fatma Mohamed Maher

Professeur-adjoint de Théâtre moderne

Département de Français

Faculté des Lettres

Université de Mansoura
Dr. Magdy Kotb Mohamed

Maître de conférences de Littérature

Département de Français

Faculté des Lettres

Université de Mansoura

r. q q

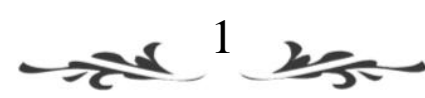

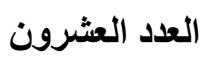


La satire de la femme dans le théâtre De Georges Courteline<smiles>C=CC=C</smiles>

بناير 9 .

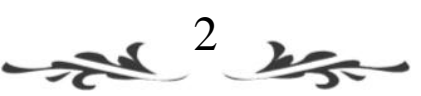

الجزء الثانى 


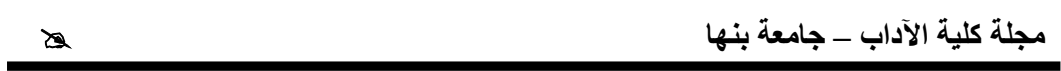

\section{INTRODUCTION}

Avant de montrer l'image de la femme aux yeux de Courteline, premièrement, on va donner une simple description de du caractère féminin en général.

La femme a un rôle très important dans la société, c'est la mère educatrice, l'autorité absolue de la famille, l'épouse qui accompagne l'homme pendant le parcours pénible de la vie. C'est l'etincelle d'amour qui transperce tous les coins de la vie conjugale et les rend douces, faciles, et confortables.

En outre de ce rôle très essentiel, si la femme travaille elle est peut être un élément efficace dans la société, elle peut participer au développement et à la renaissance de l'état. La femme est plus ouverte et plus curieuse. Elle s'intéresse à des domaines fonctionnels qui lui sont étrangers mais elle peut achever la réussite. La plupart des femmes travaillent pour aider leurs maris aux dépenses mensuelles, et une autre série travaille pour satisfaire ses ambitions personnelles.

C'est donc, n'importe quelle est la raison, un grand achèvement dur de la plupart des femmes de mettre en harmonie le travail professionnel réussi et en même temps leurs charges familiales envers leurs enfants et leurs maris.

En tout cas, les soucis familiaux pour un grand nombre des femmes ne peuvent plus être leur unique préoccupation. D'un autre côté, une grande masse des femmes croient qu'elles ont été créées seulement pour être épouses et mères et que le travail de la femme et sa participation professionnelle dans la société n'est qu'une innovation inhabituelle dans notre époque moderne. $<<$ Une femme est l'amour, la gloire et l'espérance. Aux enfants qu'elle guide $>>$ à l'homme consolé, elle élève le coeur et calme la souffrance, comme un esprit des cieux sur la terre exilée $>>1$

Et voilà, c'est l'image constituée sur la femme en général, et qui se rapproche beaucoup de la réalité. C'est la statue optimiste de la femme domestique et travailleuse qui représente les conditions normales de la vie conjugale.

Maintenant, on va aborder le côté pessimiste de la femme aux yeux de notre observateur satirique George Courteline.

L'image de la femme apparaît chez lui rarement de manière favorable. Pour lui la femme est un être stupide et ignorant. Elle peut duper l'homme habilement, gardant une

${ }^{1}$ Gérard de Nerval, http://monicalisa.over-blog.com/article-17426223-6.htm!
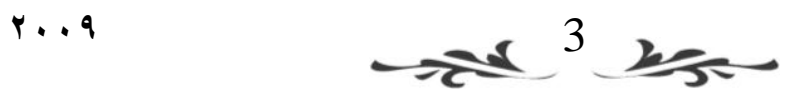
grande élégance rouée. Cet aperçu méchant qu'il a jeté sur la femme reflète la sévère expérience et les disputes qu'il a souffert avec sa première épouse, la blonde actrice Suzanne Berty et avec l'autre actrice Judith Brécourt avec laquelle il s'est lié après la mort de son épouse. Ces disputes l'obligent à travailler dans les cafés cherchant le bonheur.

$<$ II en est des femmes comme des

fous: il ne faut jamais les défier. Leur facile

menace de se jeter par la fenêtre ou d'avaler

du sublimé vaut toujours qu'on $y$ réfléchisse. $>>^{2}$

\section{a- La tromperie féminine:}

Influençant par la tromperie de son épouse et s'inspirant par celle de Jacotte la compagne qui a trompé son ami Catulle Mendès, Courteline a écrit son chef d'oeuvre Boubouroche qui a représenté à ce moment le modèle idéal de la tromperie féminine.

$<$...ayant eu l'heureuse inspiration

d'aller faire une petite visite d'amitié à une femme que j'aime profondément, j'ai eu l'agréable surprise de découvrir un visiteur, blotti dans le buffet de la cuisine... >>3

Selon Courteline, la femme est par nature infidèle et rusée. Elle peut tromper l'homme dans toutes les circonstances et dans tous les temps. La tromperie pour elle est une chose essentielle, c'est un moyen illégal pour réaliser la jouissance du plaisir.

Adèle est menteuse, c'est une femme de la petite bourgeoisie médiocre, s'attachant à la condition de la femme esclave, ce qui l'a poussé à être toujours prête à utiliser tous les moyens de malice pour se venger de son maître:

L'adultère avec André, consommé dans la jouissance du plaisir de la jeune fille amoureuse, ne lui fait pas pour autant oublier ses devoirs de maîtresse de maison, très maternelle, qui raisonne et met de l'ordre dans les bizarreries des événement.) $)^{3}$ D'une manière insolente, elle trompe Boubouroche depuis huit ans avec son amant André

\footnotetext{
${ }^{2}$ Courteline(Georges), La philosophie de George Courteline,Flammarion,Paris, 1917, p.25

3F.Turpin, Georges Courteline,Paris,Editions de la Nouvelle Revue Critique,1925,p.38
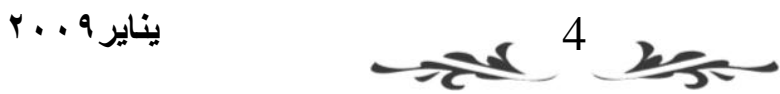

الجزء الثانى
} 
Dans cette situation, on est mal à l'aise envers Courteline qui veut insister volontairement sur la mauvaise humeur de la femme d'une manière abaissante, en exagérant d'une manière extravagante la durée de trahison, pour affirmer au public que la duperie est une tradition, par nature, féminine et mesquine.

$$
<\text { <..Oui,j'ai été huit ans ta dupe; }
$$
inexplicablement aveugle en présence de telles évidences qu'elles auraient dû me crever les yeux!...N'importe, ces temps sont finis;la canaille peut triompher, une minute vient toujours où le bon Dieu,qui est un brave homme, se met avec les honnêtes gens. $>{ }^{4}$

L'analyse comique du personnage d'Adèle renferme une langue ridicule utilisée par Courteline comme un instrument afin de provoquer le rire durable des spectateurs en suivant avec passion les événements comiques résultant de l'incroyable tromperie $d$ ' Adèle et la réaction de Boubouroche en face de ce crime.

Dans les portraits satiriques de Courteline, on remarque qu'il a souvent peint la femme comme une fille se liant d'une relation amoureuse avec un amant comme celle de son ami Catulle avec son amie Jacotte, et bien sûr influençant par ses caprices amoureuses, lui-même, avec les belles actrices avant de se marier avec l'une d'elles, la blonde Suzanne Berty.

Pierre Bornecque a montré cette remarque quand il dit: ( Il est curieux d'observer que Courteline n'a jamais peint de jeune fille \{vierge\} ni de femme seule; sans doute n'en a-t-il pas connu? En tout cas, il met en scène une jeune fille, pourvue d'un amant, ou de plusieurs, et des femmes mariées honnêtes ou trompant leur mari.) ${ }^{5}$

${ }^{3}$ http://www.evene.fr/theatre/coups-de-coeur.php?p=17, Courteline peintre de la médiocrité humaine

${ }^{4}$ Courteline, Théâtre, Boubouroche,Paris,Flammarion,1893,p.42

${ }^{5}$ Pierre Bornecque, Le Théâtre de Georges Courteline,Paris,A.G.

Nizet,1969,p.472

$r \ldots q$

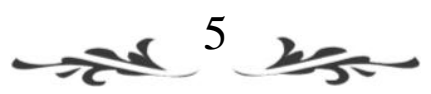


Dans L'Ecole des femmes de Molière, l'idée de la tromperie féminine est semblable à celle de Courteline. Elle devient ici une source de rire pour le public, puisque la tromperie représente pour les hommes l' être effroyable qui menace leur bonheur conjugal.

Le seigneur Arnolphe, âgé mûr, craignant toujours d'être trompé de sa femme a décidé d'épouser son orpheline, Agnès, une fille de vingt-quatre ans. II l'a éduqué dès qu'elle a eu quatre ans, il a cru qu'elle provoque la même bonté naturelle qu'elle avait eue depuis son enfance.

Arnolphe a vu que toutes les femmes sont trompeuses, l'obsession d'être un mari trompé lui a fait beaucoup de terreur, il a souhaité de fabriquer un être à sa mesure. II avait une croyance, qui annonce toujours à son esprit une foi disante, qu'il soit tranquille et confiant à son épouse, il doit éviter tous les risques dès le début du mariage et essayer mais insister à se marier, avec une femme qu'il la connaît très bien par coeur depuis l'enfance même qu'elle était mise en place de sa fille.

$<<$ Si n'être point cocu vous semble un si grand bien, Ne vous point marier en est le vrai moyen. $>6$

Donc, chez Molière qui a été trompé par sa femme, la trop jeune femme Armande ; la duperie que la femme garde provoque une distraction comique chez le public, puisque le spectateur surpris se moque de la réaction du pauvre mari soupçonneux qui est sur le point de la folie d'être un cocu. Ainsi c'est le ressort de comique dans cette pièce.

La tromperie a été incarnée donc chez Molière et Courteline de la même façon, car tous les deux ont souffert du même tourment de trahison de leurs femmes. Et pour cela on croit que leurs opinions sur la femme sont psychologiquement liées à leur condition conjugale. Ils ont été deux personnages cocus et compliqués, toutes les femmes sont à leurs égards menteuses et malignes.

${ }^{6}$ Molière,L'Ecole des Femmes,http.//www.evenue.fr/celebre/biographie/molire8.php?citations 
Cet opinion agressive résulte de leur appartenance à la classe de la bourgeoisie qui s'est été nommée à ce moment-là par le temps de l'adultère.

Mais quand on regarde la femme d'un regard un peu plus juste, certainement, on va voir que nos deux misogynes ont exagéré beaucoup dans leur description injuste de la femme. Ce n'est pas toutes les femmes trompeuses, même lorsqu'on vit dans une société où se répand la duperie; parce que s' il y existe le mauvais il est possible aussi d' y trouver le bon. On ne doit pas généraliser notre regard pessimiste, tout d'abord il faut essayer de chercher le côté optimiste .

Par exemple, Dans Le Cortège ${ }^{7}$ de Marcel Aymé, on voit un aperçu un peu clément pour la femme.

Émilie, jeune bourgeoise issue d'une bonne famille, mariée et raisonnable, a trompé son mari devant son gardien, mais finalement elle meurt après s'etre purifiée de ses péchés.

Le but de cette pièce comique de Marcel Aymé est de prouver que la femme peut atteindre la fidélité si elle veut, même lorsqu'elle était par nature trompeuse. C'est un regard un peu positif sur la femme, bien sûr, elle apparaît encore infidèle dans cette comédie mais l'auteur lui donnait la chance pour se débarrasser de ses vices. C'est le passage de la trahison à la fidèlité

Retournant une autre fois à Courteline et à une autre pièce satirique sur la duperie de la femme, c'est $L a$ conversion d'Alceste.

II est notable que Courteline a essayé de tous ses efforts de nous convaincre que la femme n'est qu'un être menteur depuis qu'elle a été créée, elle ne mérite qu' une méprise explicite de tous les hommes.

Il la considère comme une certaine chose invalide.

Courteline s'est inspiré le sujet comique de $\mathrm{La}$ Conversion d'Alceste de l'oeuvre de Molière, Le Misanthrophe. C'est est une comédie de moeurs qui porte une critique sur les défauts de la société.

\footnotetext{
${ }^{7}$ Marcel Aymé, Le Cortège,Théâtre complet 1948-1967,Paris,Gallimard,2002

${ }^{8} \mathrm{Paul}$ Carvel,http://www.dicocitations.com/citation.php?mot=Fidelite.
}

r.. q

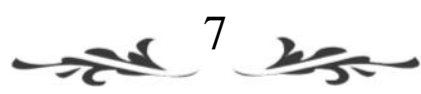


Alceste, le misanthrophe, sent une grande haine pour I'humanité, puisqu'il ne touche pas dans le monde que la flatterie et le mensonge. Il cherche l'honnêteté et la sincérité. II garde un amour extravagant pour Célimène, sa femme, et il veut quitter avec elle ce monde détestable.

Mais tristement il a été choqué dans son amour quand il connaît que ce n'est pas lui seulement qui adore Célimène qui a d'autres amoureux nombreux provoquant chez lui la jalousie et éveillant ses condamnations morales. Ses attitudes qui apparaissent trop éxagéreés aux yeux de tout le monde et même aux ceux de son intime ami Philinte, se moquant de lui toujours et encore l'infidèlité de Célimène, ont fait naître chez lui un sentiment de dépression et d'échec à réaliser toutes ses espérances pour achever la perfection morale de la vie. Finalement, il a décidé de s'éloigner de la société pour réarranger ses papiers.

C'est dans La conversion d'Alceste qu'on va observer avec Courteline la transformation surprise de la personnalité d'Alceste.

Après six mois, Alceste a retourné une autre fois, mais cette fois il a été changé complètement. II n'est pas celui qui a porté un regard négatif, pessimiste et infidèle sur I'humanité toute entière, c'est un autre Alceste qui devient heureux, optimiste et indulgent.

Il a annoncé à son ami Philinte qu'il a suivi ses conseils de se retirer un peu loin du monde détestable comme il l'a vu à ses yeux, et que ce conseil était pour lui un nouveau point de départ pour commencer une autre vie plus douce et innocente.

$<$ Oui, mon esprit baigne de nouvelle lumière

se rouvre, grâce à vous, à sa candeur première.

Je renais au bonheur d'être indulgent et bon,

Et le calme en mon coeur rentre avec le pardon. $>>^{9}$

${ }^{9}$ Courteline (Georges), Théâtre, La conversion d'Alceste,Flammarion,Paris,1905,p.173

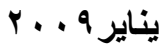

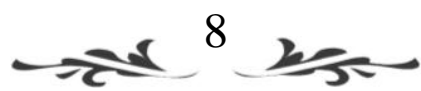

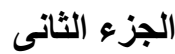


Son point de vue envers Célimène, s'est différé beaucoup de celui-là qu'il lui a conservé auparavant . Elle n'a pas été l'infidèle Célimène, mais au contraire, c'est une femme fine et gentille dont à lui son amour est devenu plus excessif . Il a fait des reproches même qu'il l'a ennuyé beaucoup avec sa bête jalousie et qu'il était un jour médiocre et méfiant devant sa pureté.

$<$ Célimène, à mes voeux souple et conciliante,

Reflet, à s'y tromper, des grâces d'Eliante,

Egayant ma maison, rassurant mon bonheur,

En toute occasion fait paraître un grand coeur. $>>^{10}$

Courteline ici provoque le rire du public, se lassant des évènements mélancoliques excités par le misanthrope Alceste, avec le changement inattendu de ses traits caractéristiques.

Mais malheureusement, ce rire ne dure pas beaucoup, puisque Courteline insiste strictement à représenter la femme comme un statut idéal de la tromperie et il a choqué Alceste d'une surprise épouvantable.

Après le sentiment du bonheur et d'amour auxquels Alceste aboutit pendant six mois d'isolement, il s'est heurté de la plus grande trahison incroyable. II a découvert qu'il était la proie de la moralité de ses éspérnces. Son ami intime Philinte essayant toujours de brûler chez lui les sentiments d'embêtement et de colère par ses caprices frivoles et ses relations amoureuses permanentes,

le trahit audacieusement avec son épouse Célimène, pour laquelle il a gardé seulement une sorte d'admiration. Célimène, l'épouse trompeuse qui n'hésite pas à aucun moment à duper son mari le cocu Alceste avec son meilleur ami Philinte.

$<<$ Certes, en m'engageant

sur la nouvelle route

${ }^{10}$ Ibid,p. 174

$r \cdot q$

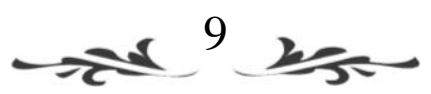


Où m'obligea mon coeur

hanté d'un dernier doute,

Je ne savais que trop où

me portaient mes pas,

Et le fossé promis au

chemin de Damas;

Mais je n'aurais pas cru, quand j'ai risqué l'épreuve,

Que les pleurs de mes yeux me fourniraient ma preuve,

Et que le crime, au seuil de ma propre maison, Me viendrait démontrer combien j'avais raison!...>> ${ }^{11}$

Alceste qui avait commencé à coexister finalement avec la société et ses défauts et avec son amour, se convaincuant qu'il a achevé le superlatif de ses espérances en faisant la paix avec le monde, a réalisé qu'il avait raison quand il a décidé de se retirer loin de cette société injuste pleine de trahison puisqu'on n'y trouve pas place pour les gens vertueux.

< Dépouillé du bonheur qui fut un temps le mien,

Maître de l'affreux droit de n'espérer plus rien, II m'est permis d'aller.----Qu'on m'y vienne poursuivre!

Traîner au fond d'un bois la tristesse de vivre, En tâchant à savoir, dans leur rivalité,

Qui, de l'homme ou du loup, l'emporte en cruauté. >> ${ }^{12}$

${ }^{11}$ Ibid p. 198

${ }^{12}$ Ibid p.199

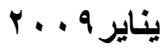

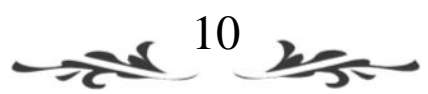

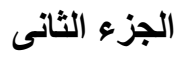


Dans cette malheureuse scène, Courteline a exprimé ses peines et ses douleurs d'amoureux, il a souffert beaucoup du cocuage au point qu'il a maudi toutes les femmes et a affirmé qu'il n'existe pas une femme sur la terre qui mérite d'être éstimée de n'importe quel homme .

Courteline se plaint de ses tortures à la langue d'Alceste dans La Conversion d'Alceste, il a donné le rôle d'incarnation du caractère du mari cocu à Alceste et d'épouse trompeuse à Célimène, mais il lui est resté le rôle le plus important, c'est l'intrigue comique du crime de la duperie reflétante de sa vie réelle.

Lorsqu' on lit les vers de cette pièce on touche absolument les traits psychologiques d'un personnage compliqué et méfiant les femmes. C'est l'auteur ( Georges Courteline).

$$
<\text { Dans la même }
$$

Conversion d'Alceste, j'ai représenté Célimène devenue la maîtresse de Philinte; niaiserie attendrissante et d'invention facile, sur laquelle je pleure aujourd'hui des flots de larmes repentantes. On parle toujours trop vite. $>>^{13}$

Dans la dernière pièce de Courteline, La Cruche, l'auteur nous a présenté la tromperie desdeux genres: la femme et l'homme. C'est à dire, qu'on va trouver dans ce drame une conséquence trompeuse; la femme trahit l'homme, l'homme trahit la femme et enfin, l'homme trahit l'homme.

Camille trompe son mari Marjevol avec Lavernite , Lavernite, le peintre, trompe Camille avec la pauvre Margot, qui était la maîtresse de son ami Lauriane.

La trahison ici est variable. Mais, la le but de cette tragi-comique n'était pas la concentration sur la tromperie, c'était, pour la première fois sur la modestie de la femme, ce qu'on va l' interpréter dans un autre point spécifique suivant.

${ }^{13}$ Georges Courteline, La philosophie de Georges Courteline, Flammarion,Paris,1917,XII, p.75 
Dans cette situation, la tromperie de la femme se subdivise en deux sortes: la tromperie volontaire,$\{$ Camille avec Lavernite\}, c'est le côté méchant de la femme.

L'honnête tromperie \{Lauriane avec Margot\}, c'est le côté sympathique envers l'homme.

< Dispute aussi dans La Cruche

entre Lavernité et Camille, qui reproche au

peintre d'avoir une autre maîtresse,puis

entre Lavernité et Lauriane à propos de

Margot. $>>^{14}$

Si on veut connaître exactement la limite extrême dans laquelle Courteline a insulté les femmes, on doit faire une tour théâtrale dans l'oeuvre de Courteline, en essayant de lire ou de voir ses pièces satiriques. En faisant ça, on remarque que la plupart de ces pièces représentent une critique piquante et une misogynie de la femme dans cette période.

L'auteur a généralisé son regard méchant sur toutes les femmes. Cet aperçu résultant, comme on a dit auparavant de ses disputes amoureuses et aussi de son appartenance à la classe de la bourgeoisie où l'adultère s'est répandue à ce moment-là.

$<<$ Les femmes, elles, trahissent les

hommes dans la proportion effroyable de

$97 \%(. .$.$) Et ça ce n'est pas une blague; c'est$

prouvé par la statistique et ratifié par la plus

élémentaire clairvoyance.>> ${ }^{15}$

Théâtre,Boubouroche,Flammarion,Paris,1900,P.36

Mais, il est bien notable que la majorité des pièces de Courteline ont abordé la tromperie du côté féminin plus que celle de l'homme, malgré que c'est l'essentiel trait caractéristique de l'homme. Cet opinion ne signifie pas que toutes les femmes sont infidèles. Mais bien sûr, comme il'ya le vice dans la société humaine il'ya aussi la vertu. $<<$ La fidélité d'une femme à un seul homme s'appelle : Vertu. La fidélité d'un homme à une seule femme s'appelle : Amour. $>>^{16}$

${ }^{14}$ Pierre Bornecque, Le Théâtre de Georges Courteline, Paris, A.G. NIZET,1969,p.344 15Courteline(Georges),

${ }^{16}$ Alexandre Weil, http://www.dicocitations.com/citation-fidelite.php 


\section{b- Une femme dépensière}

Tout d'abord, on doit jeter un coup d'oeil sur l'importance et le rôle de l'argent dans la vie de la femme. Pour la femme, l'argent est un moyen qui sert à jouir des plaisirs de la vie. C'est la seule garantie de l'avenir obscur.

La plupart des hommes font appel au secours de leurs femmes dépensières. C'est le plus souvent, la principale cause des disputes conjugales, parce qu'on trouve que la majorité des femmes règlent les dépenses mensuelles du ménage domicile. Exploitantes cette fonction familiale, elles croient qu'elles ont le droit de dépenser et d'acheter comme elles veulent, puisqu'elles possèdent un grand pouvoir d'achat.

La femme est capable de passer toute la journée en se déplaçant entre les magasins commerciaux, elle possède un grand pouvoir d'achat et elle n'hésite pas à payer des grandes sommes d'argent pour des choses inutiles qui lui plaisent, soient des habits, des accessoires, ou de maquillage. En effet, la femme peut dépenser les frais de la maison dans une promenade.

$<<$ La femme dépensière est généralement

fidèle car, très occupée par ses achats

compulsives, elle n'a pas le temps de tromper

son mari qu'avec des commerçants. $>>^{17}$

Dans cette étude, on va aborder l'analyse du caractère de la femme dépensière chez Georges Courteline.Une autre fois, Courteline a commenté la femme d'un oeil critique souvent féroce. Mais cette fois, c'est la femme dépensière. II l'a critiqué dans un de ces petits bijoux, c'est la pièce de $L a$ paix chez soi. Et comme d'habitude cette critique est venue comme résultat d'une autre expérience dure dont il a vécu encore avec son épouse Suzanne Berty.

( Courteline remit à Suzanne Berty deux cents francs pour payer un billet à ordre, puis, le coeur paisible, s'en est allé retrouver des camarades au Grand Café ou au Napolitain. Sans doute la jeune personne utilisa-t-elle l'argent à des fins qu'elle jugeait plus utiles; quoi qu'elle en soit, le billet est présenté deux jours plus tard. En vain! Du protêt, commandement, menace de saisie.

${ }^{17}$ Philippe Bouvard,http://www.dicocitations.com/citation.php?mot=depensiere

$r \cdot . q$

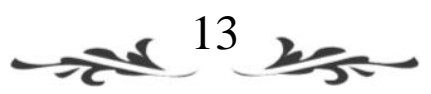

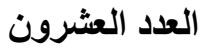


Les noirs oiseaux de procédure s'abattent, du papier timbré plein le bec; mais Courteline ne les voit pas, il n'est jamais chez lui et Suzanne, pensant non sans raison que \{ca s'arrangera\}... a pris le parti très simple de jeter au feu toute la paperasse et de n'en souffler mot à son seigneur et maître. Tant et si bien qu'un beau jour Courteline, attablé au café, voit accourir son ami Louis Marsolleau, tragique et haletant, tel un messager de Sophocle. sollicitude.

--- Qu'est- ce qui t'arrive? questionne-t-il avec

--- A moi rien, mais chez toi, ca va très mal!

--- Hein? Quoi? Que se passe-t-il?

--- Simplement ceci: I'huissier est en train de saisir tes meubles.

Effaré, Courteline se lève, court, à son domicile, arrive juste à temps pour voir enlever sa dernière chaise. $)^{18}$

Bien sûr, cette situation a offert à Courteline l'inspiration à travers laquelle il a trouvé l'occasion de citer deux des plus graves défauts de la femme.

C'est la dissipation et la négligence du ménage domestique. II a souffert de l'irresponsabilité de son épouse et son incapacité de ménager sa maison. Elle ne s'intéresse qu'à dépenser et acheter des choses inutiles en abandonnant ses charges et ses soins familiaux. A cause d'elle, il a perdu sa fortune et ses propriétés et il a été saisi par le gouvernement.

Mais qu'est ce que c'est le ménage? La plupart des femmes voient que le ménage est un devoir sacré, c'est la nature humaine pour laquelle Dieu a créé la femme. Chaque femme doit être agréable en faisant ce devoir ,en se convaincant que personne ne peut pas la remplacer et ce n'est pas une loi brutale, mais c'est une loi raisonnable. La sage femme doit être économe dans sa maison, et apprécie l'effort pénible duquel son mari souffre dans son travail, pour réserver l'alimentation et l'éducation de ses enfants

${ }^{18}$ Albert DUBEUX, La curieuse vie de Georges

Courteline,Paris,Horay,1958,p.102-103

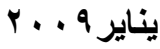

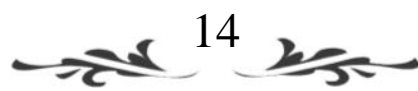

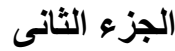


Le reste des femmes montre que le ménage est un devoir ennuyeux, c'est une sorte d'humiliation de la femme que l'on doit spécifier à des servantes. Elles protestent contre les hommes qui protègent les grands intérêts de la famille du côté matériel, et dont les soins domestiques ne sont pas sans doute, leur responsabilité. Ces femmes ne s'intéressent ni aux règles de leurs maisons ni à la surveillance de leurs enfants. Elles laissent tout aux servantes. Elles occupent seulement leurs loisirs aux centres commerciaux, acheter et payer la monnaie que leur maris s'efforcent à l'obtenir. C'est le cas de la séduisante Valentine.

$$
\text { << Un ménage est bien accordé }
$$

où les deux époux ressentent en même temps le besoin d'une querelle.>> ${ }^{19}$

Valentine, dans La paix chez soi est une femme dépensière, vaniteuse qui ne s'intéresse pas aux soins de sa maison. Son mari Trielle, journaliste, travaille dur pour réaliser ses besoins. Mais toujours, il se plaint de sa dissipation et de son échec a régler le ménage de la maison. Trielle qui aime son épouse profondément fait de son mieux autant que possible pour lui donner l'argent avec lequel elle peut acheter toutes les choses dont elle a envie. Mais la belle femme n'apprécie pas cet amour et ce sacrifice, elle se moque toujours de son mari, son insatiabilité l'a fait trop dépensière au point qu'elle peut consommer tout le frais mensuel concernant le ménage domestique, pour acheter des choses inutiles. Enfin, Le sincère mari lui a fait des reproches car elle a acheté sans payer, une lanterne à fer forgé coûtée cent cinquante francs sans le prévenir en imitant sa signature sur un chèque à payer.

Il lui a demandé de boucler le budget, et de s'intéresser un peu aux besoins de sa famille et à lui-même.

Courteline veut montrer dans cette scène la méchanceté de $\{$ Valentine\}, le caractère théâtral, \{Suzanne\}, le vrai caractère. C'est l'épouse qui se moque toujours et insolemment de son mari\{ Lâche! Lâche! Lâche! ${ }^{20}$

\footnotetext{
${ }^{19}$ JeanRostand,http://members.multimania.fr/gillou/citations/CitationsAmourMari age.htm

${ }^{20}$ Courteline, Théâtre,La paix chez soi, Paris,Flammarion,1903,p.162
}

r.. q

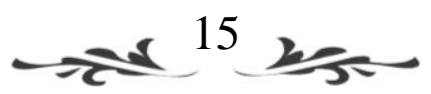


Une femme un peu maligne peut avoir tout ce qu'elle veut de l'homme \{le mari\} en exploitant sa bonté \{ Pour avoir menacé le sieur Trielle de se suicider sous ses yeux, tentant ainsi d'exploiter la tendresse de cet excellent mari. $\}^{21}$

Enfin, c'est la femme dépensière qui achète de cent cinquante francs une lanterne à fer forgé\{ $A$-t-on idée d'un tel appétit de lanterne!.... $\}^{22}$

L'auteur montre que toutes les femmes sont pareilles, elles ont un certain manque de reconnaissance, quoi que leurs maris essayent de leurs assurer le bonheur et la vie paisible.

$$
\text { < La femme ne voit jamais ce }
$$

que l'on fait pour elle, elle ne voit que ce qu'on ne fait pas. $>>^{23}$

Malgré la bêtise de Valentine, Trielle ne peut pas résister devant sa beauté, sa finesse et sa souplesse. C'est l'excitant amour qu'il lui garde. c'est le même cas de Courteline lui-même, qui était amoureux de sa belle épouse Suzanne, semblable à Valentine, et sans elle il ne peut pas vivre dans ce monde.

$\ll$ Courteline n'a peut-être tant raillé la sottise de Valentine et de ses pareilles que pour se revancher d'avoir été trop souvent sans défense devant leur séduisante puérilité. II l'indique bien nettement à Dessonnes: la conclusion de Trielle: \{ La suite au prochain numéro\} est d'une clairvoyance sans haine. Trielle est accoutumé à l'abdication: c'est à sa propre faiblesse qu'il cède.>> ${ }^{24}$

\footnotetext{
${ }^{21}$ Ibid p.162

${ }^{22}$ Ibid p. 167

${ }^{23}$ Ibid p.165

${ }^{24}$ Beatrix DUSSANE, Dieux des planches, Flammarion, 1964, p.123.
} 


\section{b- La modestie de la femme}

La plupart des hommes présentent une image de la femme très humiliée, ils croient que toutes les femmes sont les servantes de leurs maris, de leurs enfants et de leurs charges familiales. C'est l'essentielle mission pour laquelle, elles sont crééés.

$<<$ S'il l'on demande à chaque homme en particulier ce qu'il pense des femmes en général, et qu'il veuille avouer sincèrement, il dira sans doute qu'elles ne sont faites que pour nous, et qu'elles ne sont guère propres qu'a élever les enfants dans leur bas âge, et à prendre le soin du ménage $>>^{25}$

On a une autre image représentée de la femme concernant les liens conjugaux; I'homme préfère la femme faible qui l'oblige à se soumettre à ses demandes, coûte que coûte. Les larmes de la femme sont souvent, un moyen de défense contre l'injustice de l'homme, à travers desquels, celui-ci la traite doucement et avec politesse.

$<$ <...'est que le plus fort soit le maître en apparence et dépende en effet du faible; et cela non par un frivole usage de galanterie, ni par orgueilleuse générosité de protecteur, mais par une invariable loi de la nature, qui donnant à la femme plus de facilitéd'exciter les désirs qu'a l'homme de les satisfaire, fait dépendre celui-ci malgré qu'il en ait du bon plaisir de l'autre, et le contraint de chercher à son tour à lui plaire pour obtenir qu'elle consente à le laisser être le plus fort... > ${ }^{26}$

Ce qu'on a déclaré, était l'image de la femme modeste, de la part d'un grand nombre des hommes. Et maintenant, on va montrer celle-là aux yeux de Courteline, observant bien sûr, que c'est la première fois que la femme apparaît d'un aspect favorable chez lui et dont il a abordé dans sa pièce La Cruche, sympathisant avec le personnage féminin. Absolument, l'auteur a changé une partie de son opinion sur la femme à cause d'une autre expérience féminine dont il a vécu et qui a produit un grand changement dans sa vie.

\footnotetext{
${ }^{25}$ Wolfgang Leiner, Onze études sur l'image de la femme dans la littérature française du dix-septième siècle,Paris,Ed.Place,1984, p.73

${ }^{26}$ Rousseau jean-Jacques, Emile in oeuvres complètes, Ed.Gallimard, Paris, 1969,p.695
} 
Georges Courteline s'est marié avec sa seconde épouse, Marie-Jeanne, dès qu'il a passé cinquante ans, avec laquelle il a vécu heureuseument et a senti pour la première fois un état de plaisir et de pleine satisfaction. C'est elle qui s'est impressionnée quand elle a vu dans sa galerie la peinture des femmes qu'il a représenté dans les années 1890 et 1905.

< Ces petites bourgeoises 1900, insensibles, capricieuses, méchantes, vénales et jolies...dignes petites filles de Mmme Georges Dandin, suivant la formule de Jean Dutourd. $>>^{27}$

Dans La Cruche, la dernière pièce de Courteline qui était un drame sentimental, manquant de rire, il a montré le côté optimiste de la femme incarnant par Margot la maîtresse de Laurianne. Chez lui, elle a eu une place modeste souffrante de son mauvais traitement. Laurianne aime Margot mais il a insisté à l'humilier toujours en provoquant ses larmes de temps en temps devant ses amis et ses voisins. Lauriane a excité volontairement la jalousie de Margot en annonçant sa plaisance et son amour à Camille, sa voisine et la femme de Marjevol.

Le peintre Lavernite d'un âge mûr, l'ami de Lauriane et qui a caché un grand amour que Margot le mérite, a blâmé son ami à cause de sa conduite méchante envers sa douce maîtresse, il a décidé de garder Margot chez lui après l'acceptation de Lauriane qui a commencé à être lasse de cette bonne avec laquelle il a mené une vie dissolue.

$<$ Sache donc, pour ta gouverne, que j'en

plein le dos de Margot; que ma liaison avec elle a plus que suffisamment dure, mes habitudes n'étant pas de m'éterniser dans le collage.>> ${ }^{28}$

II est clair ici la différente tendance de l'auteur pour la femme. Bien sûr, il l' a présentée dans cette pièce sympathiquement en indiquant sa faiblesse devant la servitude de l'homme. II a montré que la femme ne peut pas

${ }^{27}$ Jean Dutourd:<< Le Fond et la Forme >>,N.R.F.1958,t.I,p.39.

${ }^{28}$ Courteline, Théâtre, La Cruche,Paris,Flammarion,1909,p.221
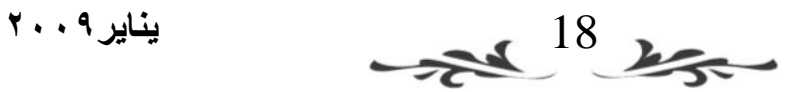
avoir ni la volonté ni la liberté devant le pouvoir masculin, spécialement quand elle est pauvre, sans argent et sans abri.

Courteline a appliqué cette croyance sur l'état de Margot qui se plaint à Lavernité qu'elle a mené une vie dure et insupportable chez Lauriane et qu'elle ne l'a jamais aimé, en indiquant son outrage dirigeant toujours vers elle exploitant son pauvreté et la vie misérable qu'elle a menée auparavant.

Et malgré tout cela elle supporte l'insolence puisqu'elle n'a pas une position préférable et plus optimiste.

< Je suis devenue sa maîtresse par la raison que je suis incapable d'être la mienne. II voulait; je ne voulais pas; à la fin, j'ai bien voulu: voilà tout le roman de mes amours qui est, en même temps, toute l'histoire de ma vie. Je ne veux pas; on veut; à la fin, je veux bien: c'est aussi simple que celà et voilà, en deux coups de crayon, le portrait de votre petite amie. Ce n'est pas de chance, d'être ainsi bâtie. Que voulezvous que j'y fasse? On ne se refait pas. Du reste, la chance et moi... >> ${ }^{29}$

Dans cette scène, on a l'impression que Courteline s'est prolongé dans son mépris de la femme. L'apparent pour nous est sa sympathie avec la femme, mais derrière celle-là , on peut empressentir son éternelle abomination. II a réussi à nous convaincre de son soutien de la femme cette fois en montrant Margot comme une femme contrainte à l'humiliation devant l'homme \{Laurianne\},

mais, le fait est qu' il a caché une autre intention; c'est que l'homme est capable d'humilier la femme s'il veut ,abondant ses sensations et ses larmes.

On a reproché à Courteline ce point de vue; parce que même dans le seul cas, où on a commencé à sentir son amour pour la femme, il a insisté encore à dessiner pour elle un portrait négatif. II a présenté Margot comme une maîtresse modeste, la cause pour laquelle, elle s'est obligée de trahir son maître Lauriane avec son ami Lavernité qui était sur le

${ }^{29}$ Ibid p.224

r. . q

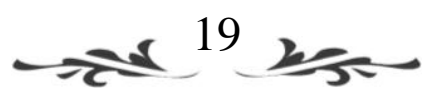


point d'avoir cinquante ans $\{$ Lavernité est le semblable de Courteline\} et qui a retourné avec elle sa jeunesse de nouveau. Enfin d'après lui, Margot est une femme bizarre dont le caractère est faible devant les hommes.

< Naturellement! J'attendais ça ! Comme toutes les femmes prises sur le fait, tu voudrais éviter une explication. Trop commode. Tu ne l'éviteras pas. A moins d'être une fille, et ce n'est pas ton cas, on ne trompe pas un homme sans motifs. Je veut savoir à quel malentendu je dois le coup de couteau qui me frappe aujourd'hui et dont je ne me remettrai jamais... >> 30

Courteline a trouvé dans le personnage de Lavernité ce qui lui a rappelé sa deuxième femme la belle; MarieJeanne. II a fait de Lavernité homme d'un âge mûr qui est tombé amoureux de la bonne Margot \{une tendre femme de vingt ans\}, espérant l'épouser, et celle là a trouvé qu'elle a pu avec lui se débarrasser enfin de sa soumission. L'auteur ici, a concentré sur le côté modeste de la femme, il a montré son besoin d'un homme qui l'aime et qui la domine sincèrement pour la sauver de celui qui l'a traitée comme une cruche.

C'est l'amour moral qui a réuni Margot et Lavernité. Malgré qu'il était sur le point de la cinquantaine, Margot a annoncé sa satisfaction, son plaisir et l'honneur qu'elle ne le mérite pas d'être la femme d'un homme comme Lavernité

< Un homme de ton âge, et du mien, demeurant toujours, quoiqu'il arrive, l'oblige du bras qui enlance, du regard qui sourit et de la bouche qui sent bie. Ceci soit dit pour elle. $>^{31}$

Dans cette situation, Courteline a protesté contre la vieillesse en donnant la confiance à lui même qu'il est capable d'aimer, de marier et de sentir le bonheur, malgré son âge mûr.

Ce n'est pas tout seulement, mais il a prouvé qu'il peut posséder une jeune femme fidèle qui l'aime beaucoup dans cette période de sa vie et ça ce qu'on appelle l' adolescence

\footnotetext{
${ }^{30}$ Ibid p.243

${ }^{31}$ Ibid p.221 
après la quarantaine \{ Voilà une enfant délicieuse, qui t'aime, te fait honneur, emplit cette maison de sa jeunesse qui l'embaume et de son rire qui l'égaye... tu ne poursuis d'autre but que le but idiot de la faire tourner en bourrique! ${ }^{32}$.

On croit que de ce point-là, Courteline a recours à incarner le statut idéal de la femme modeste à travers lequel, il a réussi de venger ses précédentes expériences amoureuses qui étaient un grand échec et à cause desquelles il a toujours attaqué la femme dans ses écrits.

$<<. . . j e$ relis les lettres des maîtresses qui m'ont trompé et des amis qui m'ont trahi; je compte mes rides du bout de mon doigt en me regardant dans la glace, et quand j'ai une minute à moi, je retouche mon testament, ou j'y ajoute un codicille. Enfin, je me distrais, quoi ! je m'amuse. $>>^{33}$

Bien sûr, l'insistance sur la servitude de l'homme à la femme était le but essentiel de Courteline dans cette pièce ,cela veut dire que comme la femme peut tromper l'homme, celui-ci a le pouvoir de l'humilier en la faisant mener une vie misérable, mais encore elle ne peut pas protester, si elle ne peut pas espérer en l'avenir. C'est le cas de Margot, quand elle a décidé de délaisser son honnêteté, qui a causé un jour la moquerie de ses amies d'elle, pour aller vivre avec Lauriane dans le collage, ne pas être comme une amante, mais comme une esclave.

$\ll<$ À l'atelier, on se moquait de moi parce que, justement, je n'avais pas d'amoureux et que, par-dessus le marché, je ne trouve rien à répondre quand on me tourne en ridicule. Je ne sais que

pleurer!... Tout cela, inévitablement, devait me livrer, un jour ou l'autre, au premier passant venu qui me tendrait les bras. Ce fut Charles qui passa... Je n'ai jamais su me défendre. Je ne peux pas; on me demande, je donne. $>>^{34}$

Margot était une ouvrière dans un atelier de couture, elle a passé un long jour de travail pénible, car elle doit être à l'atelier dès huit heures du matin. Mais le rendement est trop peu, il ne suffit pas ses besoins et son alimentation. Cependant cette pauvreté et pour la deuxième fois Margot a voulu retourner à l'atelier parce qu'elle s'est lassée de sa vie humiliée chez Lauriane. Elle a préferé la pauvreté et l'honnêteté que la richesse suivante de la servitude et l'adultère.

\footnotetext{
${ }^{32}$ Ibid p.219

${ }^{33}$ Ibid p. 214

${ }^{34}$ Ibid p.224-225
}

$r \ldots q$

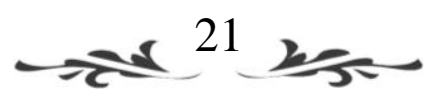


$\ll$ j'y étais encore mieux qu'ici. Je n'y gagnais pas des mille et des cent, cependant, et je peux me vanter d'en avoir boulotté, des cornets de pommes de terre frites et des moules à huit pour un sou ! sans compter qu'il y a des moments, l'hiver, après qu'on a veillé, ou c'est rudement dur de se lever à sept heures pour être à huit à l'atelier. N'importe, c'est le métier d'honnête fille qui veut ça! $>>35$

Affectée par une de ses belles femmes, Judith Brecourt, qui était une couturière dans sa jeunesse; Courteline a nommé Margot comme une ouvrière dans un atelier de couture.

À la fin de cette pièce et heureusement après la décision du mariage que Lavernité et Margot ont prit , la situation s'est changée complètement avec l'arrivee inattendue arrivée de Lauriane .

Lauriane est allé chez Lavernité pour reprendre sa maîtresse Margot afin de se marier avec elle. Margot s'est obligée d'accepter le souhait du mariage de Laurianne, abandonnant son rêve de vivre l'amour et le bonheur avec Lavernité, malgré qu'elle sache le ténébreux destin qui l'attend. Elle a convaincu Lavernité qu'il y a une grande différence sociale entre eux mêmes.

Dans cette scène tragique, on a manqué le rire. Le public s'est sympathisé avec la pauvre Margot qui a sacrifié de sa liberté et de son amour, pour revivre une autre fois dans l'obscur, dans l'espérance de trouver le plaisir de la vie plus tard.

< Nous sommes trop loin l'un de l'autre !...Un homme comme vous, mon

Dieu ! Et une pauvre malheureuse de rien du tout, comme moi...; un trottin! une midinette ! Est- ce que c'est possible, voyons? Dans six

mois je serais le boulet, dans un an je serais

l'ennemie... Je ne veux pas. $>>^{36}$

${ }^{35}$ Ibid p.223

36 lbid p.247

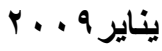

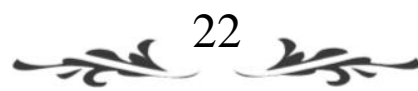

الجزء الثانى 
Georges Courteline, le génie auteur a conclu dans $\mathrm{La}$ Cruche que lorsque la femme est tombée au pouvoir de l'homme en dominant son corps et son âme exploitant sa faiblesse, mérite la pitié des gens et de la société. C'est une femme réellement modeste.

\section{2- Ridicules du couple}

\section{a- Les inconvénients du mariage:}

La dispute entre le couple est une scène de ménage passagère, c'est une forme de communication qui peut réaliser l'équilibre du mariage. La querelle pour La plupart des couples est une sorte d'affrontement qui leur donne une chance d'échanger les opinions et d'exprimer les envies. Il est nécessaire que chacun doit avoir le droit de défendre ses opinions mais sans opiniâtreté.

$\ll$ De mon point de vue, si se disputer n'est pas la meilleure des manières de communiquer, cela reste néanmoins une manière de communiquer. $>>^{37}$

Vraiment, il y a certains couples qui ne se disputent jamais, mais c'est très rare. Ces couples mènent une vie très ennuyeuse, et pour cela, ils perdent l'esprit de l'amour renouvelable et provoquante par l'excitation des querelles. Finalement, ils sont des amis mieux que des amants.

À la fin, une dispute avec une solution trouvée renforce le couple et rafraîchit la vie maritale.

Généralement, il y a quelques sujets essentiels exaltant la dispute entre les couples; ce sont le ménage, l'argent ,la jalousie, et le travail.

Notre auteur, Georges Courteline a montré les trois premiers sujets dans ses satires en penchant sur les couples et leurs problèmes conjugaux. C'est le cas dans les trois pièces qu' on va discuter, mais, tout d'abord, on doit citer la cause qui a poussé Courteline à discuter les contradictions de la vie de couple.

Après vingt ans de mariage, Courteline s'est éloigné de sa femme, spécialement, après la grande crise que les deux couples l'ont traversée et qui a duré presque dix ans.

${ }^{37}$ France - Emmanuelle,http://www.france - emmanuelle - le - blog.over blog.com/article - 29679665html 
Affectant par cette crise, Georges Courteline a négligé sa famille passant de longues nuits dans les cafés en compagnie de belles grâces, ce que l'a fait rentrer chez lui aux premières heures du matin. Son épouse ne s'est intéressée jamais du ménage de sa maison, ses premiers soucis étaient ses habits, ses toilettes et ses ornements, pour lesquels elle a dépensé de grandes sommes d'argent, ce qui a accablé son mari et en même temps, étaient pour lui l'essentielle cause qui l'a renforcée à quitter son abri conjugal, cherchant la tranquilité et le calme qui l'aident à filer ses pièces.

De là, la misérable vie conjugale de Courteline était pour lui le point de départ à travers lequel il a trouvé la chance d'aborder les querelles maritales entre les couples, en provoquant le rire des spectateurs, pour montrer sa souffrance avec sa femme, puisqu' il était un observateur qui a caché une finesse de sentiments sous une peinture méchante et féroce de ses personnages.

Dans Les Boulingrins, Monsieur Des Rillettes,est un parasite vieux et célibataire qui a été convié chez Les Boulingrins pour passer une bonne soirée et prendre le thé . Ce parasite a demandé à Félicie, la bonne, à qui il a dit des galanteries en donnant quelques sous, de lui déclarer franchement si la maison de ces Boulingrins est calme et pleine d'amour, parce qu'il a l'intention de passer chez eux les nuits les plus froides de l'hiver. Séduisante par l'argent et par l'admiration de Mr. Des Rillettes, Félicie a fait l'éloge de ses patrons en le convaincant que $\mathrm{Mr}$ et Mme Boulingrins sont deux oiseaux amoureux qui gardent une vie tranquille.

Malheureusement, le vieux parasite a découvert qu'il a été dupé par la bonne; Les Boulingrins apparaissent en faisant un grand bruit plein d'insolence, ils ont commencé à se quereller en posant chacun à l'autre des insultes haineuses.

Des bêtises se trouvant dans une maison dont on croit qu' elle est douce et dont la victime était le pauvre Mr. Des Rillettes qui a essayé de calmer la dispute et résoudre les problèmes entre les deux couples.

Mais au lieu de remercier ce malchanceux et de lui réaliser son grand espoir de jouir des soirées chaleureuses
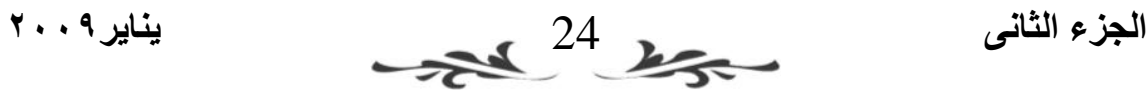
chez eux, ils ont voulu lui faire manger de la soupe empoisonnée, en lui donnant de nombreux coups et beaucoup des injures insupportables. C'est bien sûr pour eux un moyen convenable pour se débarrasser de ce parasite professionnel.

Notre démarche dans cette comédie, est de mettre en lumière I' affrontement brutal entre les deux couples qui a révélé beaucoup de sentiments de haine dont ils gardent chacun envers l'autre et qui a dominé la plupart d'événements de la pièce. Les deux mariés ont échangé toujours les insultes et les bêtises entre eux mêmes d'une manière agressive, mais comique qui montre le plus grand génie de Courteline possédant l'habileté de provoquer le rire de son public avec des personnages dont les caractères sont proches de celles avec lesquelles on vit en réalité.

Certainement, dès qu' on lit une pièce satirique de Courteline, on va sentir rapidement le rapprochement entre les personnages courtelinesques fictifs et par exemple; nos amis, nos voisins ou nos partenaires au travail. Mais ce qu'on peut ajouter est l'oeil critique de Courteline avec lequel il a peint les portraits comiques de ses personnages.

$<$ On demandait des $\{$ tranches de vie $\}$, en voilà une saignante, pantelante et amusante. Le ton est gouailleur, le style à du caractère et les personnages du tempérament, de quoi faire secouer les gens d'un rire formidable. Il a été dit également à propos de son oeuvre: \{ Vous puisez à la source inépuisable de la perversité, ou plutôt de la bêtise humaine, car ce qui fait le mal en ce monde, ce n'est pas la méchanceté, c'est la bêtise.\} $>>^{38}$

La dispute entre Mr. et Mrs. Boulingrin était un torrent d'injures et $d$ ' insolences. Toutes ses discussions ont pris un côté offensif. Ils ont recours à des insultes familières comme ; \{choléra\}, \{poison\}, \{escroc\}, \{chamelle\}, \{enfant de coquine $\}$, \{fille de voleur\}.....etc

${ }^{38}$ La presse de l'Époque,http://compagnieducadran.unblog.fr/

$r \cdot q$

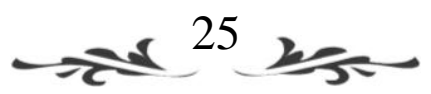


< Voilà une bougresse, fille de voleurs, voleuse elle même, qui me fait tourner en bourrique m'écorche, me larde, me fait cuir à petit feu et c'est elle qui a raison!...

Une gueuse qui me suce le sang, me ronge le cerveau, le poumon, les reins, les pieds, le foie, la rate, l'oesophage, le pancréas, le péritoine et l'intestin, et c'est elle qui a raison ! >> ${ }^{39}$

Une souffrance d'un mari mécontent de son épouse au point qu' il la rende semblable à une maladie grave qui a frappé toutes les parties de son corps, et qu' il n' a aucun espoir à l' affronter.

Ces conversations vulgaires entre les deux couples affirment la stratégie discursive que Courteline a précisé dans son théâtre. II a toujours préféré le niveau courant et la langue ordinaire dans son style comique, et c'était très clair dans la querelle déroulante entre les Boulingrins qui a représenté un phénomène habituel d'une conversation quotidienne dans la vie maritale.

$<<$ La littérature étant l'antithèse du théâtre, le théâtre, c'est l'image de la vie et dans la vie on ne parle pas comme en littérature; donc le seul fait de faire parler ses personnages littérairement suffit à les figer et à les rendre inexistants. $>>0$

Passant la parole à un autre exemple de la dispute entre les époux. Georges Courteline nous a dévoilé un autre genre des problèmes excitant la querelle conjugale.

C'est la jalousie. La jalousie est un thème répétitif de disputes, c'est un sentiment du côté du plaignant qui remue des sensations profondes envers l'accusé qui les sent bien et l'utilise pour provoquer l'amour réciproque. C'est une dispute utile pour mieux s'aimer comme l'a défini Courteline. Dans La peur des coups, le mari toujours jaloux a reproché à sa femme dès le retour de chaque bal qu' elle se fait caresser par un officier. Èvitant ces accusations pleines

\footnotetext{
${ }^{30}$ Courteline, Théâtre, Les Boulingrins,Paris,Flammarion, 1898

${ }^{40} \mathrm{http}: / /$ www.publifarum.farum.it/ezine_articles.php?art_id $=27$ 
de fureur et ce mari coléreux, l'épouse lui a donné l'adresse de cet adolescent pour venger sa dignité.

< Lui: Que je le repince, ce monsieur; qu'il me retombe jamais sous la main...Je lui flanquerai une petite leçon de savoir - vivre qui lui ôtera l'envie d'en recevoir une seconde...etc

Elle: et puis oui, je suis une honnête femme! et puis oui, tu ne seras satisfait que le jour où je serai devenue autre chose et puis oui, il m'a remis sa carte! et cette carte la voici et tu sais maintenant où le trouver et tu peux y aller tout de suite lui casser les reins à ce monsieur. $>>^{41}$

La colère du mari résultant de la jalousie exagérée dans cette pièce était très claire dans le style comique et ironique observant dans le dialogue disputable entre les deux maris, et qui est le ressort du rire des spectateurs qu' on a touché souvent chez Courteline.

< Feydeau précise ainsi sa conception linguistique au théâtre....II perçoit la vraie essence du langage théâtral et les dangers d'un discours trop respectueux des normes du code écrit. La force d'une pièce comique demeure dans le naturel du dialogue, dans la spontanéité apparente des répliques, dans l'authenticité recherchée du discours $>>42$

Dans La paix chez soi, la querelle entre les couples était un peu différente. C'est l'argent et le ménage cette fois qui ont révélé la dispute entre Valentine et Trielle. Puisque Valentine était une femme dépensière, son mari qui a fait tous ses efforts dans son travail d'écrivain en lui épargnant l'argent suffisant pour mener une bonne vie, lui a reproché sa négligence du ménage domestique et sa dissipation extravagante

Encore une fois, les deux époux ont échangé les accusations dans un discours brutal, ce qui a poussé Trielle à

${ }^{41}$ Id. La peur des coups,Paris.Flammarion,1895, p.60-61

${ }^{42} \mathrm{http}: / /$ www.publifarum.farum.it/ezine_articles.php?art_id=27 
annoncer à sa femme qu'il va mettre au point le système des amendes à chaque fois qu'elle lui dit des insolences.

< Voilà, je te le répète, cinq années que ma bonne volonté crédite ta mauvaise grâce et...que je pardonne chaque jour à la veille, dans l'espérance, toujours déçue, du lendemain...Ėnergiquement déterminé à avoir la paix chez moi et ne l'ayant pu obtenir ni par les bons procédés, ni par les moyens extrêmes, je prends le parti de l'acheter avec tes propres deniers $>>43$

La lutte entre Trielle et Valentine avait une certaine contradiction dans les sentiments. Par exemple, Trielle, était selon Courteline une exception; car c'est le seul homme qui aime sa femme sincèrement,

malgré ses inconvénients et ses bêtises, essayant de la rendre heureuse en sacrifiant de son talent comme un grand écrivain par des mauvaises oeuvres pour satisfaire au goût du public et pour lui s'assurer l'argent. \{ II est l'exemple même de l'artiste avorté, qui a perdu ses illusions et fait son métier comme une corvée pénible, sans aucune joie et en ayant conscience de la nullité de sa prose: < <...travaillant dix heures par jour à écrire des romans ineptes... $>\}^{44}$

Valentine, l'épouse capricieuse s' est moquée de son mari, au lieu d'apprécier ses efforts et ses sacrifices pour elle. Elle a essayé de lui prouver son souci du ménage et du règlement domicile. Échouant à le convaincre, Valentine pleine de colère a menacé son honnête mari de se tuer sautant par la fenêtre en l' accusant le tueur. Tous ces évènements se sont déroulés sous l'aspect d'un dialogue pénible entre les deux mariés avec des différents changements dans la présentation de la pièce commencée par la longue scène où Trielle a parlé tout seul, et suivie par les sévères scènes du début dès l'entrée de Valentine. Par exemple, lorsqu'on a commencé par le discours du monologueur Trielle, on a l'impression de ce qui va être procédé des actions.

${ }^{43}$ Id. La paix chez soi ,Paris,Flammarion,1903,p.159 - 160 - 161

${ }^{44}$ Pierre Pornecque, Le théâtre de Georges Courteline, Paris, Nizet, 1969, p.509 
On peut savoir après ça sa souffrance avec sa femme, à travers ses discussions avec son mari, et à cause d'elles le conflit entre les deux s'est provoqué . C'est la structure théâtrale de Courteline.

< Tel est donc le système d'exposition de Courteline: une grande variété dans les procédés, mais, quelle que soit la longueur de la pièce...il ne traîne jamais et informe immédiatement, où le plus vite possible, le spectateur des éléments essentiels à la compréhension de ce qui va suivre. $>>5$

\section{b- L'homme en face de la femme:}

On a discuté dans le point précédent les conflits du mariage entre les couples. La question qui est posée maintenant n'est pas celle du mariage, mais celle de la relation entre l'homme et la femme. On ne peut pas imaginer un homme sans une femme et on ne peut pas imaginer une femme sans un homme. Ils se révèlent l'un à l'autre.

$<<$ II n'y a pas de grande oeuvre dans la vie d'un

homme sans une présence féminine $>>46$

On connaît toujours que l'homme est la source de la force et du courage, il possède la sagesse et la raison dans ses décisions. L'homme a la responsabilité de trouver de la nourriture et de protéger la famille contre les dangers, tandis que la femme reste dans sa maison pour s'occuper des enfants. C'est lui dont l'identité domine celle de la femme, pour être capable d'arranger la vie maritale et familiale, cachant souvent la finesse de ses sentiments envers son épouse et ses enfants, sous une personnalité forte et brillante. À nos yeux, c'est la sculpture parfaite de l'homme. $\ll$ Guerre intestine de l'homme entre la raison et les passions... ayant l'un et l'autre, il ne peut être sans guerre, ne pouvant avoir la paix avec l'un qu'ayant guerre avec l'autre: ainsi il est toujours divisé et contraire à lui même.>> ${ }^{47}$

\footnotetext{
${ }^{45}$ Ibid p. 69

${ }^{46} \mathrm{http}: / /$ www.pingouins.com/Temoignages/HommesFemmes/body_hommesfemm es.html

${ }^{47}$ http://www.maphilo.net/citations.php?cit=6898
} 
Malheureusement, L'auteur Georges Courteline nous a présenté un autre modèle de l'homme en face de la femme, qui se diffère complètement du premier. Chez lui l'homme était un être lâche, médiocre, jaloux, avare et tourmenté toujours de l'amour qui le rend toujours aveugle devant la femme.

$\ll$ Des auteurs comme Courteline,

Pierre Wolff ont écrits des pièces fort amusantes qui supposent une vision assez pessimiste de l'homme et surtout de la femme...(Cette comédie rosse introduit) sur notre scène un type nouveau de femme, inculte, insubordonnée, volage, perverse et pourtant attirante;elle est très inférieure à l'homme dont elle partage la vie, et pourtant celui ci n'arrive pas à se déprendre d'elle:

type observé certainement par Courteline, Pierre Veber et d'autres dans leurs relations montmartoises et qui n'a pas d'équivalent dans le théâtre antérieur. $>>^{48}$

Le rentier Boubouroche dans Boubouroche qui était un bourgeois médiocre trompé depuis longtemps par son amante Adèle, impuissant d' imaginer la duperie de sa maîtresse lorsqu'un vieux et dénonciateur homme lui a révelé dans le café, après le départ de son ami Potasse, malgré qu'il a découvert Adèle avec son amant André dans son salon bourgeois.

Mais à cause de sa bonté et sa faiblesse devant son amour, la menteuse Adèle a réussi à lui persuader de son honnêteté et que cet homme n'est qu'une personne appartenant à sa famille.

« Si je n'étais une honnête femme, je ne ferais pas ce que je suis en train de faire: je ne sacrifierais pas ma vie au respect de la parole donnée, à un secret d'où dépend, seulement, l'honneur d'une autre !...

${ }^{48}$ Pierre Bornecque, Le Théâtre de Georges Courteline,Paris, Nizet, 1969,464-465
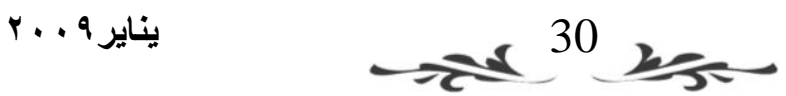

الجزء الثانى 
Sois heureux, voilà tout le mal que je te souhaite; pardonne - moi celui que j'ai pu te faire, car je ne l'ai jamais fait exprès.>> ${ }^{49}$

Après cette scène pleine d' hypocrisie qu' Adèle a présenté à Boubouroche qui était sur le point de la tuer et au lieu de demander l'indulgence de ce pauvre bourgeois, qui a aspiré lui même au pardon de sa maîtresse, cette femme trompeuse a refusé le pardon de Boubouroche qui s'est mis à la fin, à ses genoux espérant l'acceptation de son excuse.

Courteline, le peintre de la médiocrité humaine, a affirmé dans cette situation la naïveté de l'homme cocu \{ Boubouroche\} devant la bêtise et la méchanceté de la femme infidèle \{Adèle \}. II a peint un modèle de l'homme assez triste obligeant le public à sympathiser avec le personnage masculin et en même temps confirmer aux spectateurs la mesquinerie et la coquinerie de la femme.

Boubouroche est donc une pièce célèbre incarnant la bonté de l'homme soumisé à l'amour féminin, mais avec une exagération dans la description des traits caractéristiques de l'homme et de la femme qui envahissaient toute la pièce, déclarant que la femme a dominé l'homme complètement en ce temps - là.

< Boubouroche est le cocu, aveugle, incapable de croire à la trahison d'Adèle, \{ petite compagne sensée et économe, dont la fidèlité ne saurait faire question une seule minute \}; c'est $\{$ un jouet $\}$ dans ses $\{$ petites mains $\}$, il \{ sanglote, la tête dans ses jupes \}, incapable de la tuer, et acceptant ensuite son énorme mensonge: c'est le plus pathétique.>> ${ }^{50}$

Nous avons un autre exemple marquant la faiblesse masculine. C'est la lâcheté de l'homme accompagnée de la jalousie que Courteline a traitée dans une de ses comédies, La peur des coups.

Tout d'abord, on a touché dans cette pièce la jalousie du mari ,qui a fait une scène de reproches à sa femme qui a été courtisée par un militaire sensuel dans un des bals auquel ils ont assisté .

\footnotetext{
${ }^{49}$ Boubouroche,Op.cit, 1893,p.48

${ }^{50}$ Pierre Bornecque,Le Théâtre de Georges Courteline, Paris,Nizet,1969,p.471 
$<$ C'est un drôle, voilà ce que c'est ! ...Et un polisson! ...Et un sot! ... Et un gougat de la pire espèce!! ... son attitude à ton égard a été de la dernière inconvenance. II t'a fait une cour scandaleuse $>>^{51}$

II lui a demandé l'adresse de ce voyou pour le visiter et lui a donné des coups des pieds en se vengeant de lui.

L'épouse n'a pas trouvé une autre solution pour calmer la rage de son mari que de lui informer de l'adresse d'officier. Mais tandis qu'elle lui donne la carte d'adresse, elle s'assure que cet orage jaloux de son mari n'est qu'une sorte de folie qui va finir tout de suite lorsqu'il tient la carte dans ses mains.

II est très clair ici que Courteline a déclaré pour la deuxième fois la jalousie de l'homme, mais cette fois, pour interpréter un sentiment interne étant un trait essentiel qualifiant l'homme et montrant qu' à cause de l'amour, il s'est obligé d'être jaloux sur sa femme.

Or, c'est l'amour fidèle qui mouvemente les fines sensations de l'homme envers son amante et qui a tenu une place importante dans la société à ce moment là.

<< L'amour, c'est le physique, c'est l'attrait charnel,c'est le plaisir reçu et donné, c'est la jouissance réciproque... La passion, c'est l'attachement à ce plaisir et comme qui en dirait la gratitude...La jalousie, les déchirements, jusqu'au meurtre, qui en découle quelquefois, c'est le physique. C'est la représentation de l'autre auquel on tient pour son plaisir donnant ce plaisir à un autre...etc $>>^{52}$

Arrivant après la jalousie, qui a éclaté la fureur du mari contre sa femme, sa lâcheté qui le rend incapable d'affronter le séduisant capitaine qui a caressé sa maîtresse, de peur de recevoir des coups des pieds, et la femme, elle même est sûre de la poltronnerie de son mari.

« À Pâques... ou à la Trinité. Le jour où $M$. Malbrought rentrera dans le château de ses pères. $>>^{53}$

\footnotetext{
${ }^{50}$ Pierre Bornecque, Le Théâtre de Georges Courteline, Paris,Nizet,1969,p.471

${ }^{51}$ Courteine, Op.cit. La peur des coups,Paris,Flammarion,1895,p.56

${ }^{52}$ Leautaud : <<Entretiens avec Robert Mallet >>, N.R.F., 1951,P.300

${ }^{53}$ Id. La peur des coups,p.58,60
} 
La lâcheté du mari ici l' a fait forcer à cacher ses sentiments et à abandonner sa virilité et sa jalousie qui se sont disparues lorsqu' il s'est désisté de son courage et de son droit comme un mari jaloux, terrifiant de quelques attaques d'un insulteur sous prétexte du respect du costume du règlement militaire français.

$$
\text { < Moins que rien, je te dis. Le }
$$

sentiment du plus élémentaire devoir : le respect de l'uniforme français... $>>^{54}$

Bien sûr, Courteline a justifié dans cette scène de lâcheté un de ses procédés comiques et satiriques; c'est la sournoiserie des sensations qui était très claire dans le cas du mari de La peur des coups, accompagnée d'un contraste dans ses qualités éthiques. Certainement, il aime sa femme au point que cet amour le rende faible devant elle, il n' a pas supporté la voir courtisée par un autre homme, ce qui le fait devenir trop jaloux. C'est le comportement naturel de chaque homme jouissant du point d'honneur et de la force.

Mais derrière cet instinct naturel, se trouvant l' incapacité à se défendre, la manque d'audace et de la médiocrité intellectuelle. C'est la lâcheté morale. $<$ Lâches devant l'amour, vidés de volonté, de sentiment, d'idée, faibles et uniquement préoccupés de consever la femme aimée... Le contraste entre leur force physique et leur faiblesse morale, entre leur forfanterie autoritaire et leur soumission silencieuse, entre leur bêtise et l'ingéniosité de la femme, entre leur esprit et leur chair rend les amants profondément comiques.>> $>^{55}$

Le traît final caractérisant l'homme, comme l'a défini Courteline est l'avarice. On va aborder ce point là dans $L a$ paix chez soi .

\footnotetext{
${ }^{54}$ Id. La peur des coups, p.60

${ }^{55}$ Marguerite Richards, Le Comique de Courteline:thèse,Universite de NewYork,1950,p.268
} 
Valentine, l'épouse dépensière a décrit son amoureux mari de l'avarice, puisqu' il lui a imposé un système d'amendes à chaque parole blessante prononcée par elle.

En effet, Trielle n'est pas avare, mais il s'est lassé de la folie de sa femme demandant toujours de l'argent, d'une manière hardie pour obtenir ses besoins personnels. II a donc décidé de lui donner une amende sous prétexte de la punir à cause de son impolitesse.

$<$ Les cent cinquante francs

d'amende que j'ai eu le regret de t'infliger

en punition de tes écarts de language, impertinences diverses, rébellions en tout genre, etc. $>>^{56}$

L' avarice du mari était donc dans cette pièce une tentative de limiter la dissipation de la femme et de l'obliger à être économiste au règlement et au ménage domicile.

Ça veut dire que le but de Courteline dans cette comédie, n'était pas de dénoncer l'avarice de l'homme à l' égale de mépriser une attitude féminine, en trouvant une autre chance pour montrer sa misogynie contre les femmes.

$<$ Elle est souvent méchante, comme Valentine qui comble son mari de sarcasmes, se moque de lui, de son talent et dénigre son travail de journaliste... $>>57$

Ce qui est claire dans la situation précédente est la partialité de Courteline pour les hommes; il a voulu montrer une autre fois la bonté de l'homme en face la femme.

Trielle, le mari qui a aimé Valentine essayant de satisfaire tous ses désirs, s'est soumis, enfin, à ses insistances et lui a donné les cent cinquante francs, malgré son impudence et sa bêtise.

$<<$ La douceur de l'homme pour la

bête est la première manifestation de sa

supériorité sur elle.>> 58

\footnotetext{
${ }^{56}$ Courteline , La paix chez soi, p.158

${ }^{57}$ Pierre Pornecque,Op.cit, p.467

${ }^{58}$ Georges Courteline, http://www.evene.fr/tout/la-philosophie-de-georgescourteline
} 
On peut considérer La paix chez soi comme une autobiographique de Courteline dont la personnalité ressemble beaucoup à celle de Trielle. C'est une scène qui reflète l'homme devant ses faiblesses, parmi lesquelles, on touche la soumission de l'homme, excité par l'amour ardente, à la femme.

$$
<\text { <...des auteurs comme }
$$

Courteline...ont écrit des pièces fort amusantes qui supposent une vision assez pessimiste de l'homme et surtout de la femme...Cette comédie rosse introduit sur notre scène un type nouveau de femme, inculte, insubordonné, volage et pourtant attirante; elle est inférieur à l'homme

dont elle partage la vie, et pourtant celui-ci n'arrive pas à se dépendre d'elle: type observé certainement par Courteline...et d'autres dans leurs relations montmartroises et qui n'a pas d'équivalent dans le théâtre antérieur. $>>^{59}$

${ }^{59}$ Félix Gaiffe, Le Rire et la scène française, Boivin, 1931, p.246-247 


\section{CONCLUSION}

On a déjà terminé notre recherche sur la satire de la femme dans le théâtre de Courteline. Bien sûr, nous touchons la misogynie de cet auteur pour les femmes. Elles sont traitées, d'après lui, d'une manière sévère, puisqu'il était un observateur qui observe, toujours l'être humain d'un oeil critique, quelquefois, féroce.

Comme on a touché, Courteline a entouré la femme dans un portrait qui la définit trompeuse comme la duperie d'Adèle à Boubouroche, avec son amant André. Boubouroche; 1893.

Nous avons vu encore, que la femme selon Courteline est dépensière et ne s'intéresse pas au règlement de sa maison. C'est le cas de Valentine dans La paix chez soi; 1903.

Elle exploite le point de faiblesse de son mari Trielle qui l'adore essayant d'assurer pour elle le plaisir de vie.

Le troisième aperçu que l'ami, Courteline a jetè sur la femme s'est opposé, un peu, avec l'image pessimiste qu'il lui a incarné et qui s'est trouvé, souvent, dans ses écrits et ses pièces sur la femme.

Cette fois, Georges Courteline n'a pas pu cacher que les femmes, d'après lui, sont aimables et qu'il a gardé pour elles une finesse de sentiments, qu'il a essayé de la cacher dans une incarnation forte et hainee.

C'est ce qu'on a découvert dans le personnage de Margot qui est tombée victime de l'amour et esclave de son amant Laurianne. La Cruche; 1909.

Courteline n'a pas oublié d'aborder le côté de l'homme et son rapport avec la femme en penchant sur le couple et ses problèmes.

II a montré la mesquinerie et la médiocrité de Boubouroche devant la trahison d'Adèle. En même temps, l'auteur a désigné la lâcheté de Trielle avec Valentine.

On a remarqué la jalousie du marie sur son épouse dans La peur des coups; 1895.

Finalement, la soumission de l'amant Laurianne à sa maîtresse Margot était le dénouement d'un acte d'amour
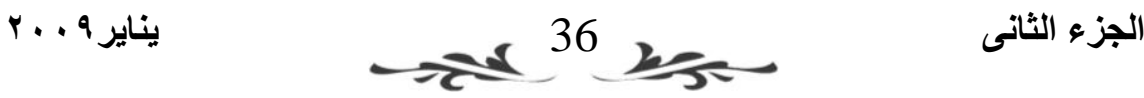
réciproque des deux amis \{ Laurianne et Lavernité $\}$ pour une seule femme\{ Margot \}.

Ce sont trois scènes de ménage qui ont occupé une grande valeur dans le théâtre féminin de Courteline. Mais la chose la plus observée dans ces actes est la domination de l'amour.

Malgré que l'auteur a présenté la femme dans ces pièces d'une manière infavorable, on ne peut pas affirmer qu'il était, purement, un détestable du personnage féminine, puisqu'il a montré, à chaque fois, la faiblesse et la bonté de l'homme, provoquant par l'amour excitée devant la force naturelle la plus effective dans le monde du mariage. C'est la force féminine. 


\section{La satire de la femme dans le théâtre De Georges Courteline}

\section{ملخص باللغة العربية :}

الهجاء الساخر للمر أة فى الكتابات المسرحية لجورعج كورتولين

يتتاول هذا البحث الأسلوب الساخرللكاتب المسرحي جورج كورثولين و سخريته من المرأة فى عصر البرجوازية الصغيرة، حيث كان هذا الكاتب

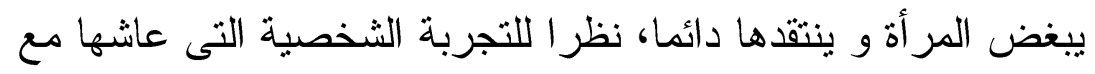
زوجته الاولى؛ الممثلة الثقر اء: سوزانى برتي.

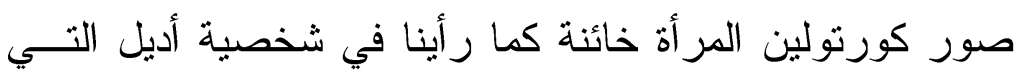

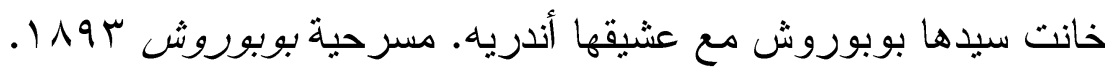

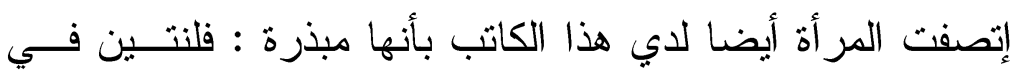

$$
\text { مسرحية السلام في بيتي س. 19. }
$$

و أخير ا كانت المرأة ذليلة و هذه هي الحالة الوحيدة التى تعاطف الته

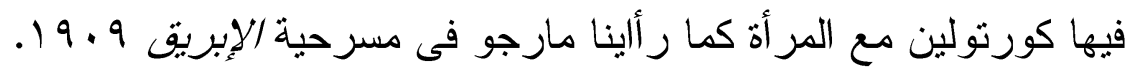

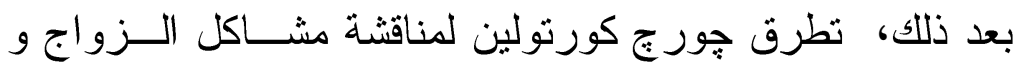

مساوئه و النزاعات الدائمة بين الزوجين موضحا ضعف الرجل و إنهياره

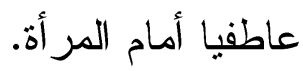




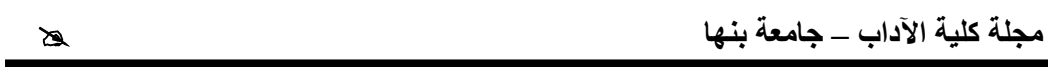

. Le Corpus

\section{Bibliographie}

- Courteline \{Georges\}, Théâtre, Ed.Flammarion, Paris, 1965,249 pages.

. Ouvrages de Georges Courteline, consacrés à la femme:

- Boubouroche, Paris, Flammarion, 1893.

- La peur des coups, Paris, Flammarion,1895.

- Les Boulingrin, Paris, Flammarion,1898.

- La paix chez soi, Paris, Flammarion,1903.

- La Conversion D'Alceste, Paris, Flammarion,1905.

- La Cruche, Paris, Flammarion,1909.

- La philosophie de Georges Courteline, Paris,

Flammarion, 1917.

- Ouvrages consacrés à Courteline;

- Pierre Bornecque, Le Théâtre de Georges Courteline, Paris, A.G.Nizet,1969,p.472.

- F. Turpin, Georges Courteline, Paris, Ed. de la Nouvelle Revue Critique,1925,p.38.

- Albert Dubeux, La curieuse vie de Georges Courteline, Paris, Horay, 1958,p.102-103.

- Béatrix Dussane, Dieux des planches,

Fammarion,1964,p.123.

- Marguerite Richards, Le comique de Courteline, thèse,Université de New.York,1950,p.268.

- Ouvrages généraux consacrés à la femme:

- Wolfgang Leiner, Onze études sur l'image de la femme dans la littérature française du dix-septième siècle, Paris, Ed.Place,1984,p.73.

- Rousseau Jean-Jacques, Emile in oeuvres complètes, Ed. Gallimard, Paris,1969,p.695.

- Jean Dutourd, Le Fond et la Forme, N.R.F.1958,P.39.

- Leautaud, Entretiens avec Robert Mallet, N.R.F,1951,P.300.

- Molière, L'école des

\section{Sitographie}

Femmes,http://www.evenue.fr/celebre/biographie/moliere-8-php?citations

- Paul Carve,http://www.dicocitations.com/citation.php/mot=Fidelite

- Gerard de Nerval,http://monicalisa.over-blog.com/article-17426223-6.html

- http://www.evene.fr/theatre/coups-de-coeur.php? $p=17$, Courteline peintre de la médiocrité humaine.

Philippe

Bouvard,http://www.dicocitations.com/citation.php?mot=depensiere 